\title{
The Processing Parameter of Folding Table
}

\author{
Junyuan Wang ${ }^{1, \text { a }}$ \\ ${ }^{1}$ School of North China Electric Power University, Baoding 071000, China \\ a261961900@qq.com
}

Keywords: folding table, processing parameter, length of slot.

\begin{abstract}
To study a folding table, through the structure of initial and final static state, determine the length of slot. Create a model on the basis of understanding the whole dynamic process and the structure of the folding table, and then determine the length of slot and the mathematical description of the edge of the table feet.
\end{abstract}

\section{Introduction}

The desktop of the folding table is round. With the activity of the hinge, the table legs can be flattened into a flat. The table legs consist of several pieces of wood. Each side of the wood is connected by a steel bar, and there is a slot on each piece of wood to ensure the freedom of sliding. ${ }^{[1]}$

In this paper, using a simple mathematical model we study the dynamic process of the folding table and the optimal design parameters. The design parameters of the table and the mathematical description of the edge of the table feet are obtained. The mathematical design of the folding table design and the edge of the table is obtained.

\section{The design parameters of the Folding table}

\subsection{The Length of the Leg}

Now we have a rectangular board size of $120 \mathrm{~cm} \times 50 \mathrm{~cm} \times 3 \mathrm{~cm}$, each pieces of wood's width is $2.5 \mathrm{~cm}$, and folded table's height is $53 \mathrm{~cm}$. We can see the radius of the folding table round is $\mathrm{r}=25 \mathrm{~cm}$, the total number of pieces of wood is $50 / 2.5=20$. Due to the table is symmetrical, it is only necessary to discuss the dynamic changes process about ten pieces of wood.

Take the round center of desktop as the origin to create Cartesian coordinate system, as shown in Figure 1:

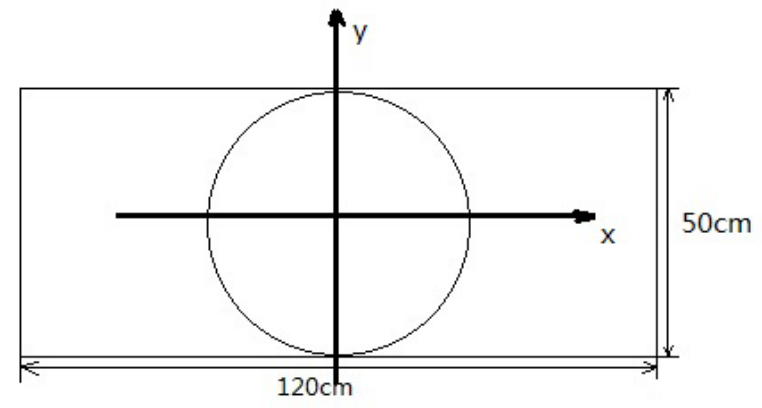

Fig.1 Desktop top view

Count the number of woods starting from the origin. Consider the woods beneath the $\mathrm{x}$-axis in Figure 1. Assume that the length of the woods are $\mathrm{Li}$, and the vertical distance between the wood and the $\mathrm{x}$-axis is qi, according to the geometric relationship, we can know:

$$
\begin{aligned}
& x^{2}+y^{2}=25^{2} \\
\mathrm{q}= & 1.25+(\mathrm{i}-1) \times 2.5
\end{aligned}
$$

By the Pythagorean Theorem:

$$
q^{2}+m^{2}=r^{2}
$$


Simultaneous equations (1) (2) (3), and solved the length of the woods of desktop semi-circular The relationship between the length of the leg and the length of the desktop is:

$$
L+m=60
$$

Table 1 The Length of the Woods

\begin{tabular}{|c|c|c|c|c|c|c|c|c|c|c|}
\hline Number & 1 & 2 & 3 & 4 & 5 & 6 & 7 & 8 & 9 & 10 \\
\hline $\begin{array}{c}\text { Length of } \\
\text { desktop / cm }\end{array}$ & 24.94 & 24.68 & 24.17 & 23.38 & 22.28 & 20.83 & 18.93 & 16.43 & 12.95 & 5.45 \\
\hline $\begin{array}{c}\text { Length of leg / } \\
\text { cm }\end{array}$ & 35.06 & 35.32 & 35.83 & 36.62 & 37.72 & 39.17 & 41.07 & 43.57 & 47.05 & 54.55 \\
\hline
\end{tabular}

Desktop’s segmentation as shown in Figure 2:

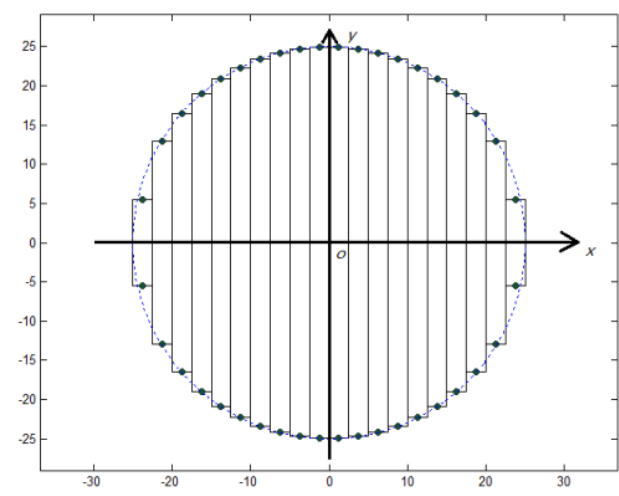

Fig. 2 The Desktop segmentation statuses

\subsection{The Length of Slot}

Considering the outermost table legs only, the lateral view of the table is shown as Figure 3.

Because folded table's height is $\mathrm{h}=53 \mathrm{~cm}, \sin \theta=(53-3) / 54.55=0.91659$

After folding the table, the longest leg is perpendicular to the ground completion of folding, the state of the table as shown in Figure 4:

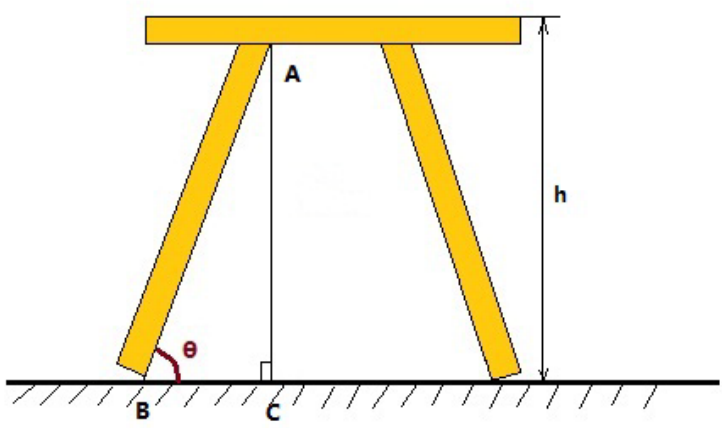

Fig.3 The lateral view of the table

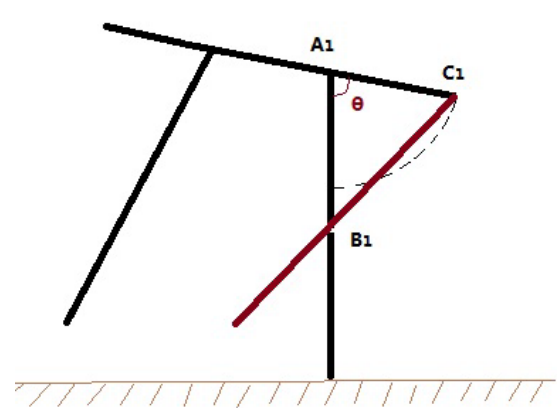

Fig.4 The state of the folded table

In the triangle $A_{1} B_{1} C_{1}$, since the steel bar connecting legs fixed to the center position in the outermost wood, we can see: $\mathrm{A}_{1} \mathrm{~B}_{1}=\mathrm{l} 10 / 2=27.275 \mathrm{~cm}$

By the cosine theorem:

$$
B_{1} C_{1}^{2}=A_{1} B_{1}^{2}+\left(m_{i}-m_{10}\right)^{2}-2 A_{1} B_{1}\left(m_{1}-m_{10}\right) \cos \theta
$$

The lengths of the slot are, as shown in Table 3:

$$
s_{1}=B_{1} C_{1}-\left(A_{1} B_{1}-\left(m_{i}-m_{10}\right)\right)
$$


Table 2 Legs Slot Length

\begin{tabular}{|c|c|c|c|c|c|c|c|c|c|c|}
\hline Number & 1 & 2 & 3 & 4 & 5 & 6 & 7 & 8 & 9 & 10 \\
\hline $\begin{array}{c}\text { The length of } \\
\text { slot / cm }\end{array}$ & 18.65 & 18.31 & 17.64 & 16.62 & 15.25 & 13.50 & 11.34 & 8.71 & 5.46 & 0 \\
\hline
\end{tabular}

\section{The Mathematical Description of the Edge of the Table Feet}

Select the shortest piece of wood to analyze the changes of table feet in the two-dimensional space. Create Cartesian coordinate system as shown in Figure 5.

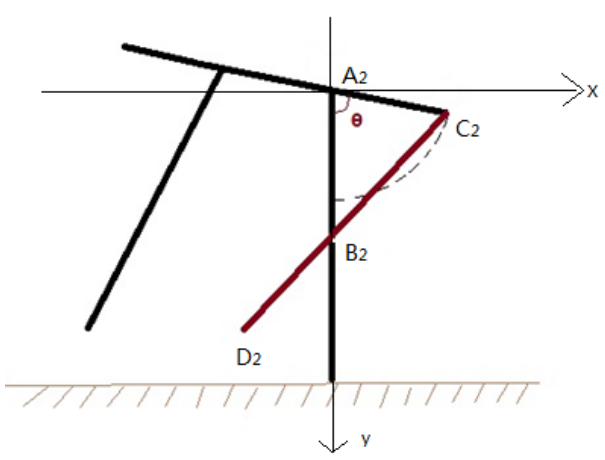

Fig. 5 The establishment of two-dimensional coordinate system

Take the round center of desktop as the origin to create three - dimensional Cartesian coordinate system. The straight line through the origin and parallel to the steel is taken as the z-axis, and positive direction is vertical paper outwards. $x$-axis and y-axis as shown in Figure 8.

Count the number of woods starting from the origin. Set the number of woods is I, and the distance from the center of the circle is $\mathrm{xi}=1.25 * \mathrm{i}$. So In the upper semicircle:

$$
\mathrm{a}_{i}=\sqrt{25^{2}-1.25^{2} \mathrm{i}^{2}}
$$

So the length of leg is $60-\mathrm{a}_{\mathrm{i}}$.

The coordinates of $\mathrm{C}_{2}$ are $\left(\mathrm{a}_{1}, \mathrm{~b}_{1}\right)$.

$$
\sin \theta=\frac{a_{1}}{60-a-5.45}, \cos \theta=\frac{b_{1}}{60-a-5.45}
$$

The coordinates of $\mathrm{C}_{2}$ are $(0,27.275)$.

Set the coordinates of $\mathrm{D}_{2}$ are $(\mathrm{x}, \mathrm{y})$.

$\mathrm{B}_{2}, \mathrm{C}_{2}, \mathrm{D}_{2}$ are in one straight line. The slope of the two-point connection is equal, so we can know:

$$
\frac{b_{1}-27.275}{a_{1}}=\frac{y-27.275}{x}
$$

The distance between the two points C2 and D2 is the length of leg that is 60-y. So we have:

$$
\left(a_{1}-x\right)^{2}+\left(b_{1}-y\right)^{2}=(60-a)^{2}
$$

From the formula (5) (6) (7) (8) we can obtain:

$$
\left\{\begin{array}{c}
\left(a_{1}-x\right)^{2}+\left(b_{1}-\frac{\left(b_{1}-27.275\right) x}{a_{1}}-27.275\right)^{2}=4225-120 \sqrt{25^{2}-1.25^{2} i^{2}}-1.25^{2} i^{2} \\
\left(a_{1}-\frac{a_{1}(y-27.275)}{b_{1}-27.275}\right)^{2}+\left(b_{1}-y\right)^{2}=4225-120 \sqrt{25^{2}-1.25^{2} i^{2}}-1.25^{2} i^{2} \\
\mathrm{z}=1.25 \mathrm{i}
\end{array}\right\}
$$

Different woods take different i values. 
According to formula (9), different woods take different i values. Draw the edge line of the table feet using MATLAB ${ }^{[2]}$, as shown in Figure 6:

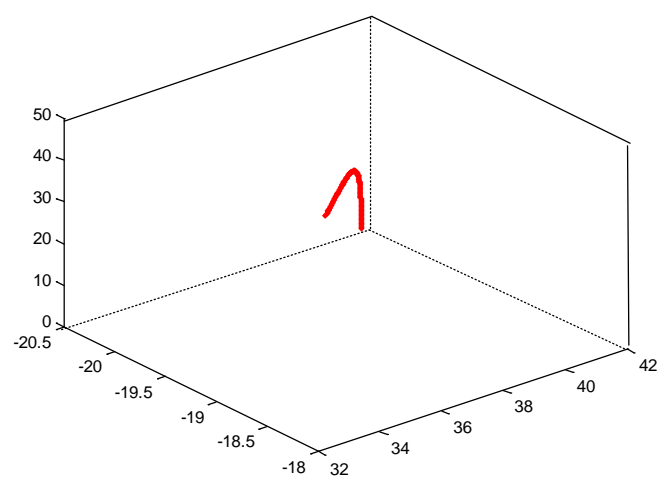

Fig. 6 The edge of the table feet

\section{Conclusions}

In this paper, the design parameters of the round folding table are studied, and the structure of the folding table is analyzed. The length of the slot is determined by the static model. First in the two-dimensional space the coordinates of the table feet are gotten. Then according to the number of woods, the coordinates of each wood in the $\mathrm{z}$ axis are gotten. And finally get the edge line of the table in the three-dimensional space. This paper provides some reference for the optimization design of flat folding table.

\section{References}

[1] Sihui Jing, Guangfang Yang, Longlong Lin, Analysis of Mathematical Model Based on Creative Flat Folding Table, J. Reform \& Openning, 9(2015) 116-119.

[2]Shan Xue, et al. MATLAB basic tutorial. Beijing: Nation Tsinghua University Press, 2015, p. 208-213. 